\title{
Software Development Effort and Cost Estimation: Neuro-Fuzzy Model
}

\author{
${ }^{1}$ Divya Kashyap ${ }^{2}$ Ashish Tripathi ${ }^{3}$ Prof. A. K. Misra \\ I,2,3 (CSED, MNNIT, Allahabad, India)
}

\begin{abstract}
Software development effort and cost prediction is one of the important activities in software project management. Accuracy in prediction is a challenge for software developers. There are many models exists that defines a relationship between size and effort. Cost of developing a project increases with complexity of project accuracy predictions are strongly required during the early stages of project development. Because data and info available at the starting phases of project is not complete, not consistent and not even certain. An objective of the software engineering community is to develop a useful model that define the development life-cycle and accurately predict the cost of developing a software product.

In this paper we discuss Neuro-Fuzzy model deals with this situation. Neuro-Fuzzy models are the combination of Artificial Neural Network and Fuzzy Logic. Artificial Neural Network has the ability to learn from previous data. It model complex relationships between both independent variables (cost drivers) and dependent variables (effort). Fuzzy logic simulates the human behavior and reasoning. Fussy logic is basically used in situation where decision making is very difficult and conditions are not clearly defined. Facts that may be dismissed are focused in this technique.
\end{abstract}

Keywords: Neural Network, Fuzzy Logic, Artificial Neural Network.

\section{Introduction}

Software cost estimation has been an important but difficult task since the beginning of the computer era in the 1940s. As software applications have grown in size and importance, the need for accuracy in software cost estimating has grown too. Software development effort-estimation is the process to predict the most realistic use of effort required to develop or maintain software based on incomplete, uncertain

and/or noisy input. Cost estimation is sometimes referred as

"parametric estimation" because cost-estimation accuracy demands understanding the relationships among scores of discrete parameters that can affect the outcome of software projects. Some major problems encountered during cost estimation process are: (i) factor that affect the cost are non-linear in nature so it is very difficult to establish a mapping between these factors and output metrics, (ii) data present at the starting phases are incomplete and imprecise so measurement of metrics are difficult, (iii) there are many models available for estimation purpose but the problem is how to determine which model is useful in which situation,and (iv) difficulty to use algorithmic model(numerical data)[1] and non-algorithmic model[2,5] together. To overcome these problems, Neuro-Fuzzy model is proposed. This model is a combination of Artificial Neural Network and Fuzzy Logic. It shows better result than the existing individual algorithmic and non-algorithmic cost estimation models.

\section{Neural Network}

Neural network is a powerful class of model which includes many diverse models and approaches. Information processing by neural network is inspired by the way biological nervous system, like Brain, process information. It has a novel structure for processing information. To solve a specific problem, a large number of neurons (i.e. densely interconnected processing elements) working in unison. Specific application such as pattern recognition or data classification, through a learning process is configured by neural network. Neural network learn by examples. They do not follow algorithmic approach.

\section{Fuzzy Logic}

In the race for developing sophisticated control system, Fuzzy Logic has become one of the most noteworthy technologies. Fuzzy logic is a control system methodology that solves problems in a very large domain ranging from simple, small to large, networked or workstation based control system. Two logic supports either true or false (also known as truth value). In Fuzzy logic an intermediate truth value, such as may be true is also possible. Many applications have reported successful achievements of using Fuzzy logic. Fuzzy logic has made it easier in understanding image applications. Fuzzy logic has ability to behave like human mind and apply effectively the logics that are approximate rather than exact. 


\section{Solving Approach}

Xishi [6] et al. proposed a Neuro-Fuzzy model for cost estimation which is modified in the current paper. It is applied on various estimation problems for accuracy. The Neuro-Fuzzy model contributes

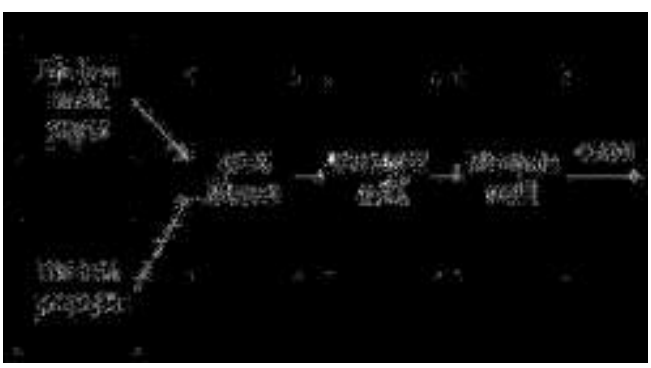

Figure 1: Proposed Neuro-Fuzzy model for cost estimation

with algorithmic model like COCOMO, Function Point. In [6], Xishi uses three components in soft computing framework for software cost estimation. Three components are: Preprocessing Neuro-Fuzzy inference system

(PNFIS), Neuro-Fuzzy bank (NFB), Algorithmic models. Model

proposed in this paper(shown in Figure 1) has certain modifications over Xishi's soft computing model. In the proposed Neuro-Fuzzy model for software development effort estimation, 22 software cost drivers are used as initial inputs. Cost drivers affect the value of software

development cost. These cost drivers include 17 Effort multipliers $\left(\mathrm{EM}_{1} \ldots \mathrm{EM}_{17}\right)$ and 5 scale factors $\left(\mathrm{SF}_{1} \ldots \mathrm{SF}_{5}\right)$ [2] (shown in Figure 2).

These cost drivers are used as input for expert judgment and analogy system. Based on their experience, experts give their judgment about the degree of the drivers for the next level i.e. Non-algorithmic system. Experts with special and particular experience are required in this model. Data sources are: (i)

Industrial project data: 6 projects, (ii) COCOMO'81 dataset: 63 projects.

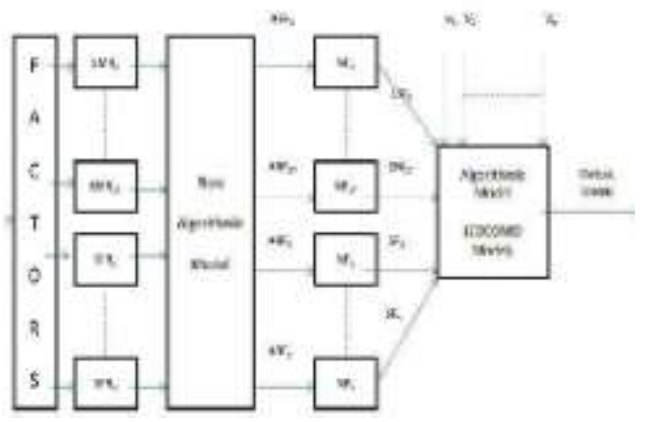

Figure 2: Proposed Neuro-Fuzzy architecture for cost estimation

Neuro-Fuzzy system maps degree of drivers to Adjustment rating factors (ARFs). Output ARFs are passed through the Neuro-Fuzzy system which produces corresponding numerical values known as multiplier for inputs to the algorithmic values. Artificial Neural Network and fuzzy logic is applied to the ARFs. Fuzzy logic system is constructed on the basis of fuzzy net, fuzzy rules and fuzzy influence. Output ARFs and values $\left(\mathrm{V}_{1} \ldots \mathrm{V}_{\mathrm{n}}\right)$ which are used originally in the COCOMO [1] are now passed to the COCOMO algorithmic model that finally concludes the estimation process. COCOMO uses the mathematical formula for cost estimation process. ARF improves the accuracy of the outcome of algorithmic COCOMO model. This is because the proposed model uses the capabilities of artificial neural network, fuzzy logic's "if-then", expert judgment and analogy which improve the accuracy of the existing model.

\section{V. $\quad$ Exprimental Result}

The results of the models are evaluated by using Mean Magnitude of Relative Error (MMRE), PRED (Prediction percentage), RMSE (Root Mean Square Error), Root Mean Square Standardized Error (RMSSE) etc. [5, 8] Results obtained with the ANN models indicate that the performance of such a model mainly depends on its architecture and parameter settings, and relying on empirical rules to determine these settings is not the optimal approach. A summary of Neural Network studies [7] is tabulated in Table 1.

In [5], Ihtiram Raza Khan et. al. propose model with MMRE less than 15\% in the case of Function point and improvement of accuracy in larger proportion in the case of COCOMO. In [8], Parvinder S. S. et. al. 
calculates the output of Neuro-Fuzzy system has the lowest MMRE and RMSSE values i.e. 0.11943 and 7.0731 respectively with comparison to other models.

\begin{tabular}{|l|l|}
\hline Study & Result \\
\hline Venkatachalam & Promising \\
\hline Wittig \&Finnie & MMRE $=17 \%$ \\
\hline Jorgenson & MMRE $=100 \%$ \\
\hline Serluca & MMRE $=76 \%$ \\
\hline Samson et al. & MMRE $=428 \%$ \\
\hline Samivasan \& Fisher & MMRE $=70 \%$ \\
\hline Hughes & MMRE $=55 \%$ \\
\hline
\end{tabular}

Table 1: Result of Neural Network studies [7]

Table 1 summarizes the results of the existing models and in the proposed model, the expert judgment, analogy and inference with Neuro-Fuzzy model are combined that can improve the accuracy percentage of cost estimation. In this paper, all issues and their respective solutions are considered; which may affect the estimation process and not discussed in the existing models.

\section{Future Work}

In last decades, most of the researchers mainly focused to find the cause of software project failure. Inaccurate estimation at the early stages is the main reason for this failure. So focus on estimation models is very important to achieve reliable and accurate estimation. In current paper, most of the related existing algorithmic and non-algorithmic estimation models are discussed. Project managers use models according to the situation and requirement. Performance of these models depends on complexity of the project, experience of the expert, development method and so on. To improve the accuracy we combine the Neuro-Fuzzy framework with existing estimation model COCOMO. The benefit of using artificial Neural Network is that it has the ability to learn from the previously completed projects. Advantage of applying

Neuro-Fuzzy framework is its interpretability because fuzzy logic is used. Fuzzy logic simulates the user's idea in estimation. The proposed model can be used in various estimation problems because, in proposed model, algorithmic and non-algorithmic; both models are used. Future work will be to apply proposed model to other estimation models like function point, SLIM, SEER-SEM etc. and also try to develop a tool for this purpose.

\section{References}

[1] Boehm. "Software Engineering Economics", Prentice Hall, 1981

[2] Idri, A., S. Mbarki, et al. "Validating and understanding software cost estimation models based on neural networks". Information and CommunicationTechnologies: From Theory to Applications, 2004. Proceedings. 2004 International Conference on, 2004.

[3] M. Jorgensen, B. Boehm, et al. "Software Development Effort Estimation: Formal Models or Expert Judgement?" Software, IEEE 26(2): 14-19, 2009.

[4] Vahid Khatibi, Dayang N. A. Jawawi "Software cost Estimation Methods: A Review", 2010-2011. Journal of Emerging Trends in Computing and Information Sciences on 2010-2011.

[5] Mr. Ihtiram Raza Khan, Prof Afshar Alam, Ms. HumaAnwar. "Efficient Software Cost Estimation using Neuro- Fuzzy Technique", National Conference onRecent Developments in Computing and its application, August 2009.

[6] Huang X, Ho D, Ren J, Capretz L, “ A Soft Computing Framework for Software Effort Estimation”Soft Computing Journal, Springer, available at www.springeronline.com, 2005.

[7] Ali Idri, Taghi M. Khoshgoftaar and Alain Abran. "Can Neural Networks be easily Interpreted in Software Cost Cost Estimation?", 2002 World Congress on Computational Intelligence, Honolulu, Hawaii, May 12-17, 2002.

[8] Parvinder S. Sandhu, Porush Bassi and Amanpreet Singh Brar. "Software Effort Estimation Using Soft Computing Techniques", World Academy of Science, Engineering and Technology, 46, 2008. 